\title{
SIMULATION OF IRREGULAR WAVES IN A NUMERICAL WAVE TANK
}

\author{
Li Zhi-Fu \\ Shi YuYun \\ Ren HuiLong ${ }^{a}$ \\ Li Hui \\ Muhammad Aqeel Ashraf ${ }^{\mathrm{b}, \mathrm{c}}$ \\ a) College of Shipbuilding Engineering, Harbin Engineering University, Harbin Heilongjiang. China \\ b) Department of Geology, Faculty of Science, University of Malaya, Kuala Lumpur, Malaysia \\ c) Faculty of Science \& Natural Resources, Universiti Malaysia Sabah, 88400 Kota Kinabalu, Sabah, Malaysia
}

\begin{abstract}
The time domain boundary element method was utilized to simulate the propagation of the irregular waves in a numerical wave tank. The problem was solved in a time-marching scheme, upon the irregular waves being fed through the inflow boundary, in which the theoretical solution was obtained from the wave energy spectrum. The open boundary condition was modeled by the multi transmitting formula (MTF), in which the phase velocity was calculated according to the Sommerfeld's condition. The velocity potential and wave elevation were directly obtained by integrating the free surface condition twice, with respect to time. The accuracy of the developed numerical scheme was verified by simulating the propagation of irregular waves. The numerical results show good agreements with the analytical solutions, which prove that the proposed scheme is a promising way to the simulation of wave-body interactions.
\end{abstract}

Keywords: open boundary condition, Multi transmitting formula, Irregular waves, Boundary element method, Numerical wave tank

\section{INTRODUCTION}

The Rankine panel method is a main way to simulate the interactions between floating structures and waves. The method has many merits in the simulation of nonlinear problems [1], ship motions with forward problems [5], varying bottom of ocean floor simulation [8] and hydroelasticity response of marine vessels [4].

However, if the Rankine panel method is utilized, it's necessary to truncate the computation domain into finite to save computer cost. And then the non-reflection boundary condition is needed to guarantee the accurate of the simulation. The Sommerfeld-Orlanski's condition have been widely used by researchers [6, 7], but it cannot give a good result for irregular waves simulation. Marching with linear solutions method is used by Liu $[10,11]$, but the computation cost is huge. The piston-like active wave absorber is also used in common [2,3], but it is hard to be applied in 3D problems. Another frequently used method is the artificial beach [9], but some panels will be wasted. In addition to the above, the multi-transmitting formula method is also widely used by researchers $[14,15]$, but the phase velocity has to be set before the simulation, which is a limitation to the simulation of irregular waves.
The aim of this paper are, therefore, (1) to develop a numerical scheme to simulate the propagation of irregular waves in a numerical wave tank, (2) to find an efficient nonreflection open boundary condition for the Rankine panel method. The accuracy of the numerical scheme is verified by the comparison of analytical solutions with the numerical ones.

\section{MATHEMATICAL FORMULATION}

The Cartesian coordinate system is adopted to describe the fluid motions, with its origin in the undisturbed free surface, $\mathrm{x}$-axis positive to the wave propagation, and $\mathrm{z}$-axis positive in the opposite direction of gravity, shown in Fig.1. The fluid is assumed to be ideal, incompressible, its motion is irrotational, and the surface tension is ignored. Then the velocity field can be described by the velocity potential.

$$
\boldsymbol{V}(\vec{X})=\left(\frac{\partial \phi}{\partial x}, \frac{\partial \phi}{\partial y}, \frac{\partial \phi}{\partial z}\right)
$$

Due to the mass conservation, the fluid should satisfy the Laplace equation in the whole fluid domain.

$$
\nabla^{2} \phi=0
$$




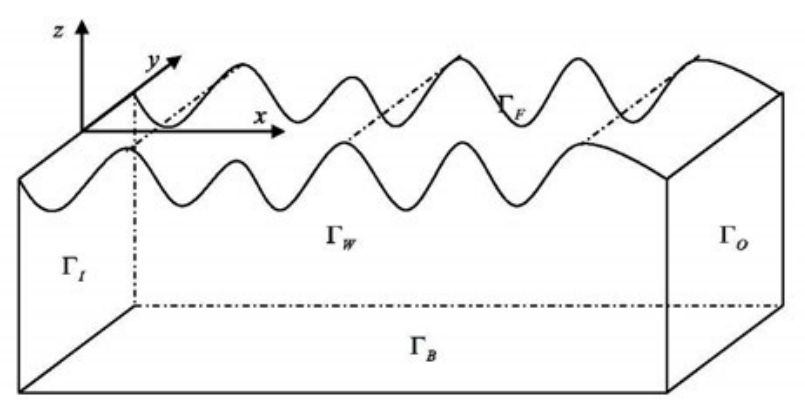

Fig.1. Coordinates system and computation domain

The kinematic boundary condition specifies that the free surface moves with the fluid particles, and the free surface kinematic boundary condition can be written as

$$
\frac{\partial \varsigma}{\partial t}+\frac{\partial \phi}{\partial x} \frac{\partial \varsigma}{\partial x}+\frac{\partial \phi}{\partial y} \frac{\partial \varsigma}{\partial y}-\frac{\partial \phi}{\partial z}=0, \text { on } \Gamma_{F}
$$

The fluid pressure for the irrotational flow is determined by the Bernoulli's equation, and assuming the pressure on the free surface is constant, then the dynamic free surface condition can be obtained,

$$
\frac{\partial \phi}{\partial t}+\frac{1}{2} \nabla \phi \cdot \nabla \phi+g \varsigma=0, \text { on } \Gamma_{F}
$$

The linear free surface conditions can be obtained from Eqn.(3) and Eqn.(4) by using a perturbation procedure. The kinematic and dynamic conditions are,

$$
\begin{aligned}
& \frac{\partial \varsigma}{\partial t}-\frac{\partial \phi}{\partial z}=0, \text { on } z=0 \\
& \text { and } \\
& \frac{\partial \phi}{\partial t}+g \varsigma=0, \text { on } z=0
\end{aligned}
$$

By substituting (5) into (6) we can get

$$
\frac{\partial^{2} \phi}{\partial t^{2}}+g \frac{\partial \phi}{\partial z}=0, \text { on } z=0
$$

Integrating (7) twice with respect to time, and using the trapezoidal numerical integral method as well as the initial conditions. Then at each time step, the velocity potential on the free surface can be written as

$$
\phi(p,(n+1) \cdot \Delta t)=\phi(p, n \cdot \Delta t)-g \cdot \Delta t^{2} \cdot \sum_{i=1}^{n} \frac{\partial \phi(p, i \cdot \Delta t)}{\partial z}
$$

By using the same method, we can obtain the wave elevation in the time marching scheme.

On the lateral boundary, the rigid wall condition will be used, for the simulated waves are unidirectional,

$$
\frac{\partial \phi}{\partial y}=0 \text {, on } z=0
$$

and the non-penetrating condition at the bottom boundary is

$$
\frac{\partial \phi}{\partial z}=0, \text { on } \Gamma_{B}
$$

To enclose the problem, the fluid domain should be truncated at some distance from the region of interest. Then an artificial boundary should be adopted, as will be described in the following sections.

$$
\phi=\phi_{M T F}, \text { on } \Gamma_{O}
$$

\section{OPEN BOUNDARY CONDITION}

Open boundary modeling is of great importance in the simulation of irregular waves in the time domain. Various approaches have been developed to accommodate the elliptical problem. In this research, the MTF was combined with the Sommerfeld's condition, in which the Sommerfeld's condition is used to evaluate the wave phase velocity, and for the short waves the damping zone method will be distributed near the open boundary, but with a very short damping length $\left(\lambda_{\text {min }}\right)$.

The MTF for the non-reflecting boundary conditions was first proposed by Liao [10], via a direct simulation of one way wave propagation using a space-time extrapolation by an artificial wave speed. According to the theory of Liao, for the $\mathrm{N}$-order MTF, the velocity potential on the open boundary can be written as

$$
\phi((p+1) \Delta t, 0)=\sum_{j=1}^{N}(-1)^{j+1} C_{j}^{N} \cdot \phi\left((p+1-j) \Delta t,-j \cdot c_{a} \cdot \Delta t\right)
$$

where $\mathrm{C}^{\mathrm{n}}$ is the binominal coefficients

$$
C_{j}^{n}=\frac{n !}{(n-j) ! j !}
$$

The Sommerfeld's radiation condition [13] is known as follows:

$$
\frac{\partial \phi}{\partial t}+c \frac{\partial \phi}{\partial n}=0 \text {, on } \Gamma_{O}
$$

Combination of the dynamic free surface condition (6) and the Sommerfeld's condition (14) yields

$$
c=\frac{g \zeta}{\phi_{n}}
$$

Therefore, the artificial phase velocity can be calculated in the time marching scheme according to (15). In this paper, the 2-order MTF approximation formula will be used.

$$
\phi(t+\Delta t, x)=2 \phi\left(t, x-c_{a} \cdot \Delta t\right)-\phi\left(t-\Delta t, x-2 c_{a} \cdot \Delta t\right)
$$

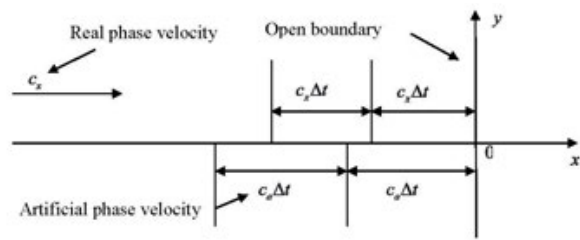

Fig.2. Sketch of multi-transmitting formula 


\section{NUMERICAL METHODS}

Various numerical approaches can be used to solve this initial boundary value problem mentioned above, among which the boundary element method has been widely used. The direct boundary integral equation to solve the prescribed boundary problem is derived using the Green's second identity.

$$
2 \pi \varphi(p, t+\Delta t)=\iint_{S}\left[G(p, q) \frac{\partial}{\partial n_{q}} \varphi(q, t+\Delta t)-\varphi(q, t+\Delta t) \frac{\partial}{\partial n_{q}} G(p, q)\right] \mathrm{ds}_{q}
$$

Two symmetry planes are utilized to model the half domain and exclude the bottom boundary. The resulting Green function is

$$
G(p, q)=\frac{1}{R_{1}}+\frac{1}{R_{2}}+\frac{1}{R_{3}}+\frac{1}{R_{4}}
$$

Where $\mathrm{p}(\mathrm{x}, \mathrm{y}, \mathrm{z})$ is the field point and $\mathrm{q}(\zeta, \eta, \varsigma)$ is the source point, and

$$
\begin{gathered}
R_{1}=\sqrt{(x-\xi)^{2}+(y-\eta)^{2}+(z-\varsigma)^{2}} \\
R_{2}=\sqrt{(x-\xi)^{2}+(y+\eta)^{2}+(z-\varsigma)^{2}} \\
R_{3}=\sqrt{(x-\xi)^{2}+(y+\eta)^{2}+(z+\varsigma+2 d)^{2}} \\
R_{4}=\sqrt{(x-\xi)^{2}+(y-\eta)^{2}+(z+\varsigma+2 d)^{2}}
\end{gathered}
$$

\section{EXPERIMENT RESULTS}

\section{SIMULATION OF REGULAR WAVES}

Before the simulation of irregular waves, it's necessary to verify the numerical schemes, by simulating the propagation of the regular waves. For the regular wave simulation, the boundary value problem was solved in the time marching scheme. The total simulation time was, and the time step was , and six panels for each wavelength. The range of wavelength is from to . The velocity potential time history of the partial record point was compared with the theoretical input waves, shown in Fig.3. The numerical results are in good agreement with the analytical ones, which indicates that the present model of the open boundary works well for the non-reflection condition.

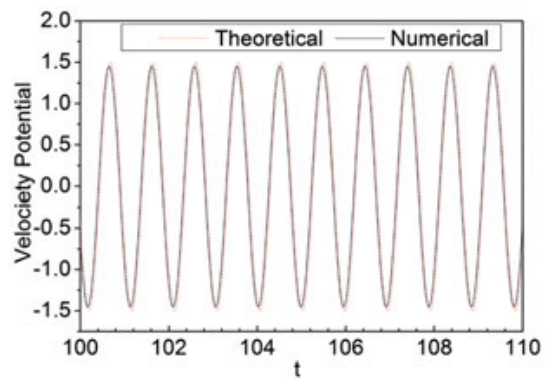

(a) wavelength $\lambda=1.45 \mathrm{~m}$

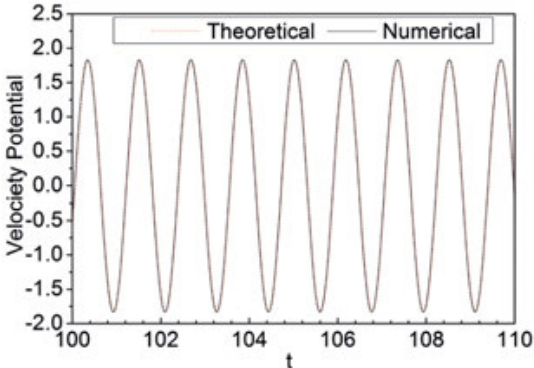

(b) wavelength $\lambda=2.13 \mathrm{~m}$

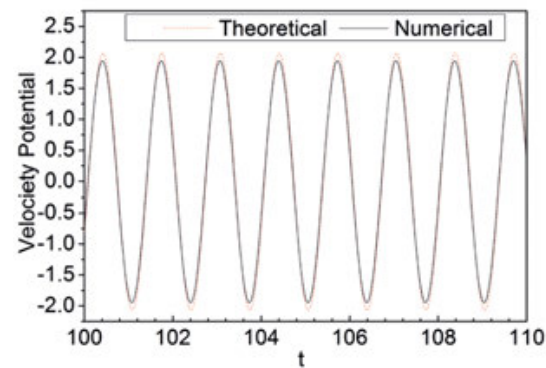

(c) wavelength $\lambda=2.75 \mathrm{~m}$



(d) wavelength $\lambda=4.50 \mathrm{~m}$

Fig.3. Time history of the simulated regular waves at the middle point

\section{SIMULATION OF IRREGULAR WAVES}

The dimensions of the numerical wave tank was $10 \mathrm{~m} \times 2 \mathrm{~m} \times 2 \mathrm{~m}$, six elements were distributed for one wavelength, and the time increment was set for $\mathrm{T} / 40$, , The total simulation time was $200 \mathrm{~s}$. The theoretical solutions were obtained from the wave energy spectrum, with the wave frequency domain $0.5 \mathrm{rad} / \mathrm{s} \sim 4.5 \mathrm{rad} / \mathrm{s}$. The full records of the wave velocity potential were measured at the longitudinal tank center, which were compared with the theoretical waves. And the values of simulated waves agree fairly well with the theoretical solutions, shown in Fig.4.

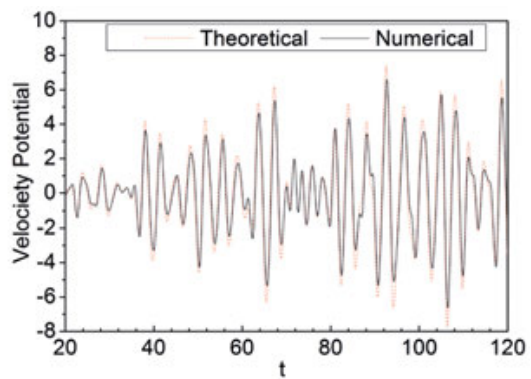

(a) record point in the center 


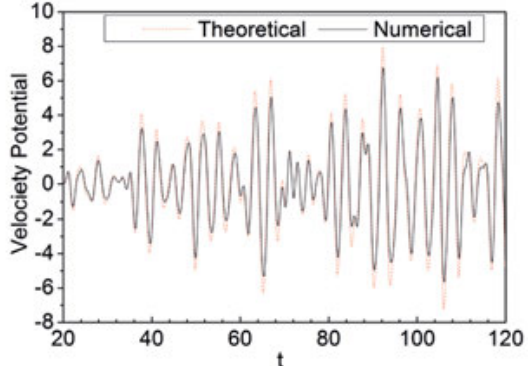

(b) record point near $\Gamma_{1}$

Fig.4. Time history of the simulated irregular waves

\section{CONCLUSIONS}

In the present work, a time domain numerical program was implemented to the simulation of irregular wave propagations, and the boundary value problem was solved in the time marching procedure.

The non-reflection open boundary condition was modeled by the MTF, which can let the wave pass totally if the artificial phase velocity is similar to the real phase velocity. Through the combination of the Sommerfeld's condition with the kinematic free surface condition, the formula used to evaluate the phase velocity was derived. And then the wave simulation results were compared with the theoretical ones, including regular waves and irregular waves, respectively. The wave simulation results show excellent agreement with the theoretical values. After a series of numerical experiments, it has proven that the proposed non-reflection open boundary model can be applied to the simulation of wave-body interactions.

Further study may be done to predict the linear or nonlinear wave-body interactions using the proposed non-reflection open boundary condition.

\section{ACKNOWLEDGMENT}

The authors gratefully acknowledge the financial support provided by the National Natural Science Foundation of China (Grant No. 51079034), and by the National Basic Research Program of China(Grant No. 2011CB013703).

\section{REFERENCE}

1. Bai, W. and R.E. Taylor, Higher-order boundary element simulation of fully nonlinear wave radiation by oscillating vertical cylinders. Applied Ocean Research, 2006. 28(4): p. 247-265.

2. Brandini, C. and S. Grilli, Modeling of freak wave generation in a 3D-NWT. 2001.

3. Clément, A., Coupling of two absorbing boundary conditions for 2D time-domain simulations of free surface gravity waves. Journal of Computational Physics, 1996. 126(1): p. 139-151.
4. Das, S. and K.F. Cheung, Hydroelasticity of marine vessels advancing in a seaway. Journal of Fluids and Structures, 2012. 34(0): p. 271-290.

5. He, G. and M. Kashiwagi, A time-domain higher-order boundary element method for 3D forward-speed radiation and diffraction problems. Journal of Marine Science and Technology, 2013. 19(2): p. 228-244.

6. Isaacson, M. and K.F. Cheung, Second order wave diffraction around two-dimensional bodies by timedomain method. Applied Ocean Research, 1991. 13(4): p. 175-186.

7. Jagannathan, S., Non-linear free surface flows and an application of the Orlanski boundary condition. International journal for numerical methods in fluids, 1988. 8(9): p. 1051-1070.

8. Kim, T. and Y. Kim, Numerical analysis on floatingbody motion responses in arbitrary bathymetry. Ocean Engineering, 2013. 62: p. 123-139.

9. Kim, Y., D.C. Kring, and P.D. Sclavounos, Linear and nonlinear interactions of surface waves with bodies by a three-dimensional Rankine panel method. Applied Ocean Research, 1997. 19(5-6): p. 235-249.

10. Liao, Z.-P., Extrapolation non-reflecting boundary conditions. Wave Motion, 1996. 24(2): p. 117-138.

11. Liu, S.K. and A.D. Papanikolaou, Time-domain hybrid method for simulating large amplitude motions of ships advancing in waves. International Journal of Naval Architecture and Ocean Engineering, 2011. 3(1): p. 72-79.

12. Liu, S.K., A. Papanikolaou, and G. Zaraphonitis, Prediction of added resistance of ships in waves. Ocean Engineering, 2011. 38(4): p. 641-650.

13. Orlanski, I., A simple boundary condition for unbounded hyperbolic flows. Journal of computational physics, 1976. 21(3): p. 251-269.

14. Xu, G. and W.-y. Duan, Time-domain simulation for water wave radiation by floating structures (Part A). Journal of Marine Science and Application, 2008. 7: p. 226-235.

15. Zhang, W.D.T., Non-reflecting simulation for fullynonlinear irregular wave radiation. 24th International Workshop on Water Waves and Floating Bodies, 2009. 
CONTACT WITH AUTHOR

\author{
Li Zhi-Fu \\ lizhifu@hrbeu.edu.cn \\ Ren HuiLong \\ College of Shipbuilding Engineering \\ Harbin Engineering University \\ Harbin Heilongjiang \\ 150001 China \\ Shi YuYun \\ Li Hui, \\ Muhammad Aqeel Ashraf \\ Department of Geology \\ Faculty of Science \\ University of Malaya \\ 50603 Kuala Lumpur \\ Malaysia
}

